\title{
Bone Mineralization in an Osteogenesis Imperfecta Mouse Model Studied by Small-Angle X-ray Scattering
}

\author{
P. Fratzl, ${ }^{\star \ddagger}$ O. Paris, ${ }^{\ddagger}$ K. Klaushofer, ${ }^{\star}$ and W. J. Landis ${ }^{\S}$ \\ ${ }^{*}$ Ludwig Boltzmann Institute for Osteology, 4th Medical Department, Hanusch Hospital, Vienna, Austria; ${ }^{*}$ Solid-State Physics Institute, \\ University of Vienna, Austria; and ${ }^{\S}$ Children’s Hospital and Harvard Medical School, Boston Massachusetts 02115
}

\begin{abstract}
We have studied the size and orientation of mineral crystals in cortical bone of oim/oim mice, which are known to produce only $\alpha 1$ (I) collagen homotrimers and which may serve as a model for human osteogenesis imperfecta. Long bones (femur and tibia) from young (5 wk old) oim/oim mice and from unaffected heterozygous counterparts were investigated by small-angle x-ray scattering (SAXS), which is sensitive to structures smaller than $50 \mathrm{~nm}$. Mineral crystals were compared in terms of their thickness and their alignment with respect to the long bone axis. While electron microscopic tomography has recently shown the existence of large mineral blocks (with all dimensions typically exceeding $50 \mathrm{~nm}$ ) in mineralized tendons of oim/oim mice, SAXS revealed a family of thin, possibly needle-like, crystals in cortical bone. These crystals were similar in shape to those observed previously in normal mice, but they were thinner and less well aligned in oim/oim mice relative to heterozygotes. Moreover, the crystal thickness and their alignment with the bone axis were more variable in oim/oim bone, with a close correlation $(r=0.94, P<0.001)$ between the two parameters. The presence of smaller crystals with more variable alignment in corticalis of oim/oim mice may contribute to the brittleness of their bone, similar to that of human osteogenesis imperfecta. (J. Clin. Invest. 1996. 97:396-402.) Key words: osteogenesis imperfecta $\bullet$ bone mineral $\bullet$ x-ray scattering $\bullet$ bone biomechanics $\bullet$ collagen
\end{abstract}

\section{Introduction}

Osteogenesis imperfecta $(\mathrm{OI})^{1}$ is a genetically determined disease of connective tissue, clinically characterized by bone fragility. The disease is generally attributable to mutations of type I collagen (1-3). The origin of the brittleness of OI bone is not yet quite clear although structural abnormalities in the collagen/mineral composite of the bone, such as hypo- or hypermineralized regions (4), changes in collagen cross-links and mineral crystallinity (5), and abnormal collagen fibril diame-

Address correspondence to Dr. Peter Fratzl, Institut für Festkörperphysik der Universität Wien, Strudlhofgasse 4, A-1090 Wien, Austria. Phone: 1-31367-3242; FAX: 1-3100183; E-mail: fratzl@pap.univie.ac.at

Received for publication 18 May 1995 and accepted in revised form 29 September 1995.

1. Abbreviations used in this paper: OI, ostogenesis imperfecta; SAXS, small-angle $x$-ray scattering.

J. Clin. Invest.

(C) The American Society for Clinical Investigation, Inc.

0021-9738/96/01/396/07 \$2.00

Volume 97, Number 2, January 1996, 396-402 ters $(6,7)$, have recently been suggested as possible sources for the absence of tissue integrity.

Investigations of a structural basis for the brittleness in OI bone are complicated by a great variability of possible mutations of the collagen gene (1-3), leading to a broad range of phenotypes. Recently, however, a mouse model (oim), having a well-defined genetic mutation, has become available for study (8). The mouse produces $\alpha 1$ (I) collagen homotrimers and has an increased bone brittleness (8). The absence of the $\alpha 2$ (I) chain may mimic the situation in some human patients with OI type I. Collagen fibrils with abnormal molecular packing were found in the tail tendon of the homozygous oim/oim mutant (B. Brodsky, personal communication) and both small and unusually large (dimensions exceeding $50 \mathrm{~nm}$ ), block-like mineral crystals were detected in the Achilles tendons which mineralized in this animal.

Here we present the first structural study of the collagen/ mineral composite in compact bone of the oim/oim mouse using small-angle $\mathrm{x}$-ray scattering (SAXS). This technique is sensitive to structures smaller than $50 \mathrm{~nm}$ and has recently been used successfully to characterize the typical mineral crystals comprising thick untreated or resin-embedded samples of compact bone from humans and various animals including mice. Although the method is indirect, it provides quantitative information about average crystal size, shape, orientation and arrangement within a collagen/mineral composite (15). These parameters have also been suggested as critical to the mechanical strength and stability of a calcified tissue (16). A previous study of normal mice has shown, in corticalis from the midshaft region of the long bones, elongated mineral crystals principally oriented parallel to the major bone axis (12). This alignment was undetectable in bone from embryos but became apparent during the first weeks after birth. The average thickness of the earliest measurable crystals was also found to be $<2 \mathrm{~nm}$ and increased to $\sim 3 \mathrm{~nm}$ shortly after birth (12). In the present study, we use SAXS to determine alignment and thickness of the bone mineral crystals in a young oim/oim mouse mutant and its heterozygous counterpart, which did not express the disease.

\section{Methods}

A homozygous oim/oim mouse mutant having defective pro $\alpha 2(\mathrm{I})$ synthesis and its heterozygous littermate that did not show the disease were sacrificed at the age of five weeks. Both animals were approximately the same weight. While the genetic defect in the oim/oim mouse is similar to some cases of human OI type I, there is a more severe effect on the phenotype in oim/oim. Indeed, the long bones obtained from the oim/oim individual had a characteristically bent and distorted shape with severe cortical thinning as well as common fractures. Immediately on dissection, small segments of the midshaft (diaphyseal regions) of femurs and tibiae were treated by anhydrous means with ethylene glycol and Cellosolve and embedded in epon 
resin. The preparation is known to maintain the mineral phases of the tissues and details of the methods are given elsewhere (17). Longitudinal sections of the bones were cut and ground to a thickness of 200 $\mu \mathrm{m}$ (13). The sections were mounted in a SAXS camera perpendicular to the beam path of $\mathrm{x}$-rays and positioned using microcontrollers. For each sample, SAXS spectra were measured at several positions in the corticalis within the respective mid-shaft regions. SAXS measurements were performed using a $12 \mathrm{~kW}$ rotating anode generator operated with $\mathrm{CuK} \alpha$ radiation (x-ray wavelength $\lambda=0.154 \mathrm{~nm}$ ), an evacuated pinhole camera (sample to detector distance $1 \mathrm{~m}$ ) and a two-dimensional position-sensitive proportional counter (Siemens, A. G., Karlsruhe, Germany). All spectra were corrected for background scattering from the pinholes.

Typical two-dimensional spectra are shown in Fig. 1. The color coding indicates the intensity of the radiation scattered around the primary beam that is transmitted through the specimen. The primary beam is stopped before entering the detector (this is visible as a small disk in the center of the patterns in Fig. 1). The scattering signal itself is attributable to the marked difference in electron density between the mineral crystals and the organic substance of the tissue under examination. On the basis of previous studies, the SAXS signal from normal mouse bone $(12,15)$ can be described by the predominance of very long (that is, longer than $\sim 50 \mathrm{~nm}$ ) needle-shaped crystals inside a collagen matrix. The relation between the shape of the SAXS pattern and the orientation of those mineral crystals is shown in Fig. 2, where the thick arrow corresponds to the main axis of the long bones (horizontal in Fig. 1). One or a number of needle-shaped mineral crystals mutually parallel and parallel to the bone axis generates a disk-shaped SAXS pattern that is perpendicular to the needle direction (case I in Fig. 2). If some crystals are rotated with respect to the bone axis direction, their SAXS pattern rotates correspondingly. This implies that for randomly oriented crystals the SAXS-pattern is spherical (case III in Fig. 2). The data shown in Fig. 1 are planar sections through the SAXS patterns indicated in Fig. 2, the plane of section being perpendicular to the incoming primary $\mathrm{x}$-ray beam. By rotating the specimen with respect to the incoming $\mathrm{x}$-ray beam, one can show that the full three-dimensional SAXS pattern from mouse femur or tibia has cylindrical symmetry around the main bone axis, that is, the horizontal line in Fig. 1. A comparison with Fig. 2 then indicates that both patterns in Fig. 1 correspond to the intermediate case II in Fig. 2, where the crystals are generally oriented parallel to the bone axis. The two patterns in Fig. 1 are different in the degree of the resultant ellipsoid eccentricity, much larger in the heterozygote than in the homozygote. The data lead immediately to the qualitative conclusion that the mineral crystals are better aligned in the heterozygote. For a more quantitative evaluation, it is possible to determine a function $\mathrm{h}(\psi)$, which describes the probability that a mineral crystal points in a given direction tilted by an angle $\psi$ with respect to the main axis of the long bone (18). The relevant mathematical formulae are given in the appendix (equations A1 to A5).

Other quantities that may be derived from the SAXS patterns are a mean crystal thickness $T$ and a shape function $G(x)$ (12-14). To obtain these parameters one computes the spherically averaged SAXS spectrum, which is the function $\mathrm{J}(\mathrm{q})$ defined in eq. A2. In a mathematical procedure described elsewhere (15), the total volume $\mathrm{V}$ of all crystals (having at least one dimension smaller than $50 \mathrm{~nm}$ ) and their total surface $\mathrm{S}$ may be determined, yielding a parameter $\mathrm{T}=4 \mathrm{~V} / \mathrm{S}$. $\mathrm{T}$ can be interpreted as a typical crystal thickness. Indeed, when the mineral crystals are taken to have typical dimensions of $\mathrm{a}, \mathrm{b}$, and $\mathrm{c}$, with $\mathrm{c}$ much larger than the other two (needle-like crystals), then the thickness $\mathrm{T}$ is an average of a and $\mathrm{b}: T=2 a b /(a+b)$. The function $G(x)=x^{2} J(x / T) / J_{\mathrm{o}}$ has also been defined earlier (14), where the constant $\mathrm{J}_{\mathrm{o}}$ is chosen in such a way that $\int_{0}^{\infty} G(x) d x=1$. The advantage of defining this rescaled function $\mathrm{G}(\mathrm{x})$ is that it becomes independent of the typical crystal thickness $\mathrm{T}$, and it reflects only the shape and arrangement of the crystals in different samples (14).

\section{Results}

Cortical bone samples from the midshaft region of the femurs and tibiae from a 5-wk-old oim/oim mouse and its heterozy-

\section{+loim}

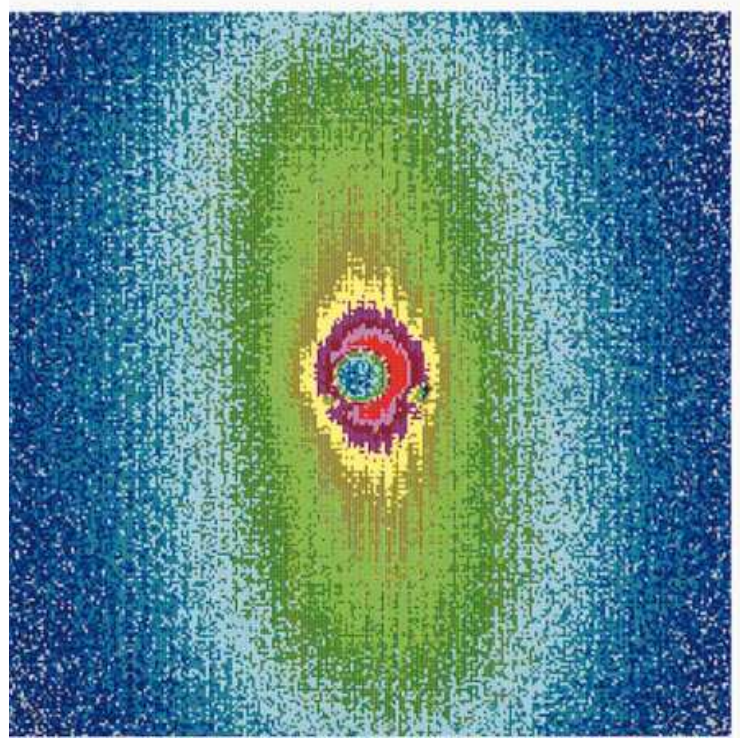

\section{oim/oim}

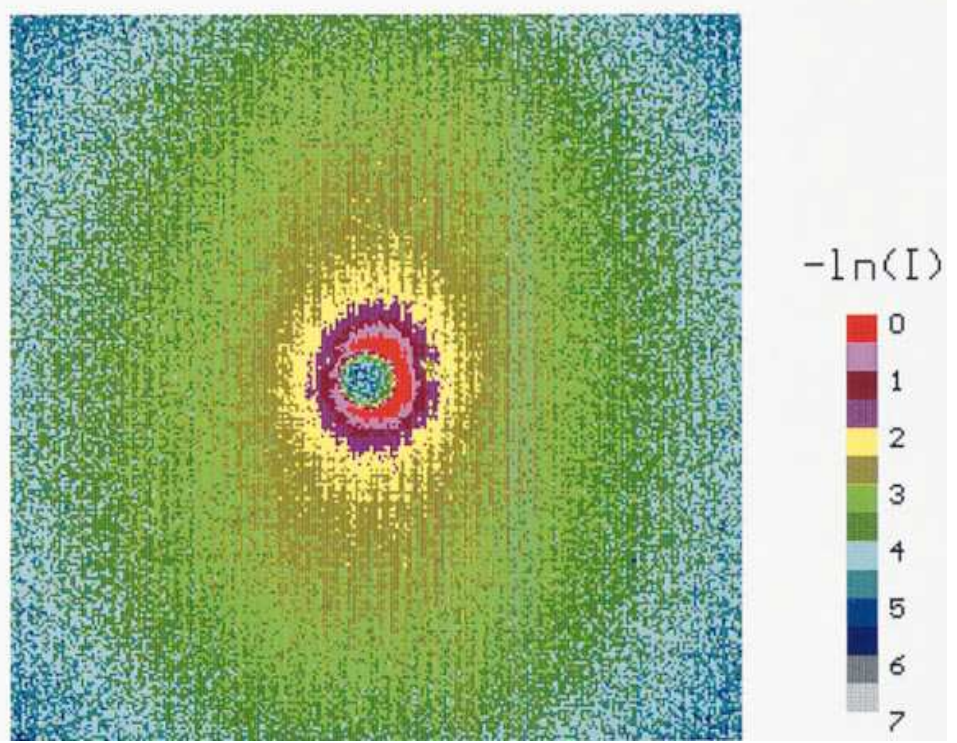

Figure 1. Two-dimensional SAXS pattern from corticalis in the midshaft region of the femur from an oim/oim mouse and its heterozygous littermate. The direction of the primary x-ray beam is perpendicular to the plane of the drawings. The axis of the long bone corresponds to the horizontal direction of the patterns. The scattering intensity is represented by a color code on a logarithmic scale. The SAXS patterns are represented within a square of scattering vectors $\mathbf{q}$ between -1 and $+1 \mathrm{~nm}^{-1}$ in both horizontal and vertical direction. The circle in the center results from the primary $\mathrm{x}$-ray beam stop. 


\section{Orientation of mineral crystals}

\section{corresponding}

SAXS pattern

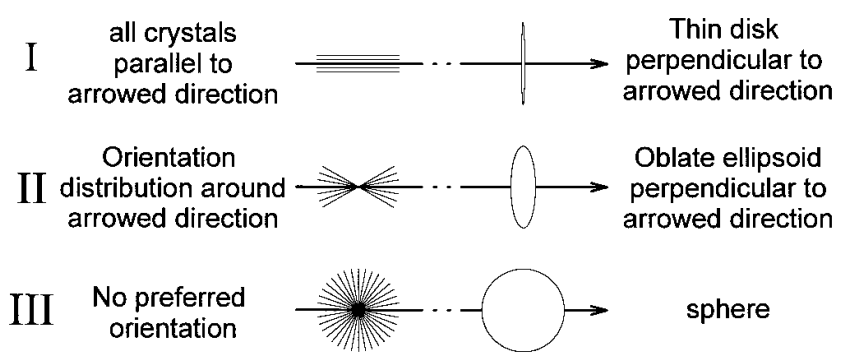

Figure 2. Schematic representation of SAXS patterns originating from elongated mineral crystals inside collagen fibrils. In $I$ all the crystals within the specimen volume illuminated by the $\mathrm{x}$-ray beam $\left(\sim 0.5 \mathrm{~mm}^{3}\right)$ are parallel, in $I I$ they are tilted by some amount and in III they are randomly oriented. The SAXS measurements shown in Fig. 1 correspond to planar sections through the three dimensional SAXS patterns as schematically shown on the right side of this figure, where the thick horizontal arrow indicates the direction of the main axis of the long bones (horizontal in Fig. 1). It should be noted, that the orientation information is an average through the whole specimen region illuminated by the $\mathrm{x}$-ray beam and that it cannot be distinguished whether the tilting of the mineral crystals occurs within the same collagen fibril, or whether whole fibrils are tilted one with respect to the other (the crystals inside a given fibril being parallel).

gous counterpart were investigated by SAXS. Fig. 1 shows, as an example, the spectra from one femur. For the heterozygote (Fig. 1, left), the SAXS pattern has the typical anisotropy already described in earlier experiments on normal mice (12). Here the elongated crystals are predominantly oriented parallel to the main bone axis (horizontal in Fig. 1). In contrast, the data for the oim/oim mutant (Fig. 1, right) are much closer to isotropic. This indicates increased orientational disorder of the collagen/mineral composite for this OI bone.

To quantitate this observation, the method described in equations A1 to A5 was used to determine $\mathrm{h}(\psi)$, the fraction of mineral crystals pointing in a given direction tilted by an angle $\psi$ with respect to the main bone axis. Fig. 3 gives the result for a typical measurement inside each bone sample (two femurs

\section{+loim oim/oim}

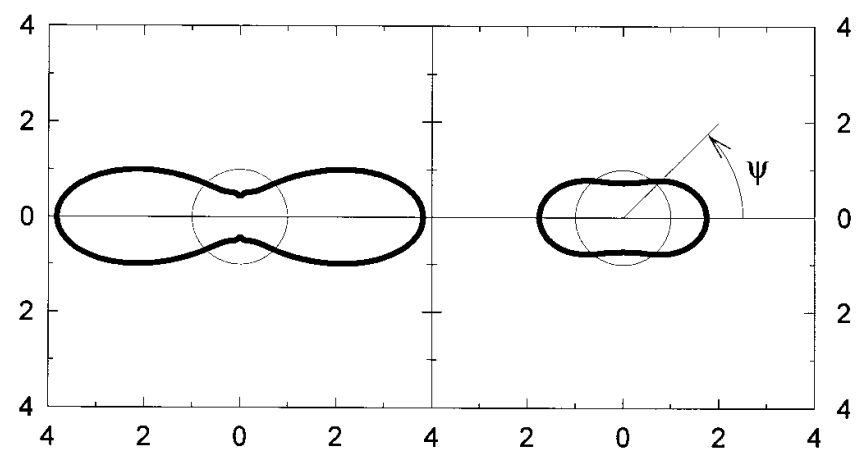

Figure 4. Polar representation of the function $\mathrm{h}(\psi)$ for femur 1 (see Fig. 3). The distance of the bold line tracing to the center of the graph corresponds to the value of $\mathrm{h}(\psi)$ at the angle $\psi$ as indicated. A random orientation of needle-like mineral crystals would produce a circle of radius 1 (shown by the thin central circle). The horizontal direction of the plots corresponds to the long bone axis. Note that the crystal distribution (represented by the bold line) is more elongated in this direction for bone from the heterozygous mouse than that for the oim/oim animal.

and two tibiae in the oim/oim and the heterozygote). The function $\mathrm{h}(\psi)$ would be a constant equal to 1 for a complete random orientation of the crystals. All curves in Fig. 3 are peaked at $\psi=0$, a result which indicates a predominant orientation of the crystals along the main bone axis. The peak height is more variable in the oim/oim mouse and is also generally smaller than in the heterozygote. Measurements at other locations of the femurs and the tibiae gave curves typically in the range of variation shown in Fig. 3. There was no essential difference between the bones in this aspect of results. Additionally, the curve marked "Femur 1" in Fig. 3 is plotted in a polar representation (Fig. 4), which can be understood as a section through the three-dimensional distribution of the crystal orientations. In the case of completely random orientation of bone crystals, this distribution would be spherically shaped and, hence, the section would be a circle as indicated by the thin line in Fig. 4. If all crystals were oriented perfectly parallel to the bone axis,

\section{+loim oim/oim}

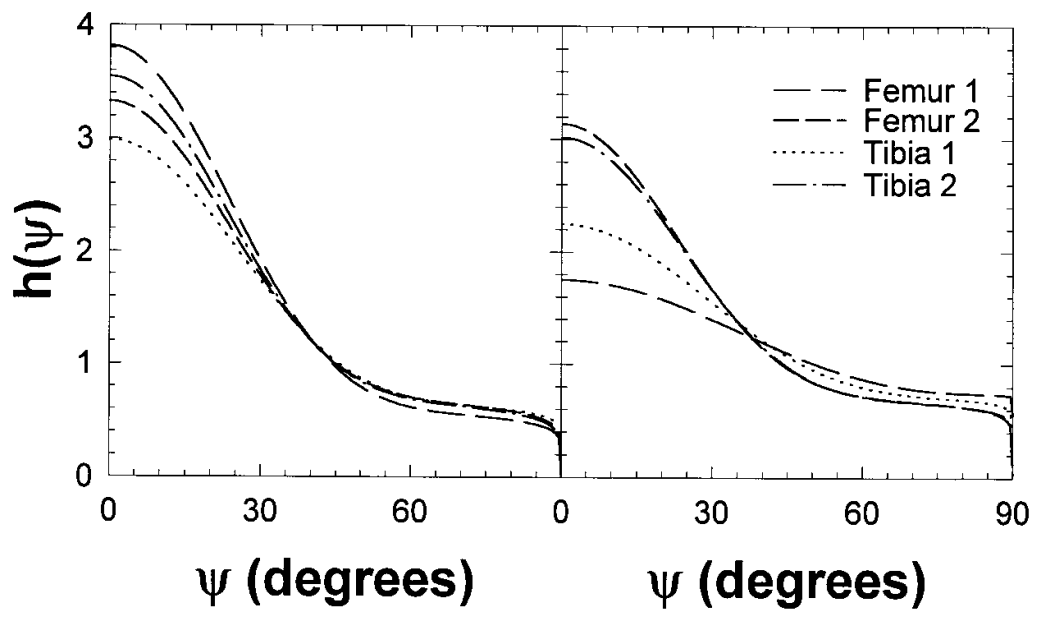

Figure 3. Probability h $(\psi)$ that a needle-shaped mineral crystal points in a given direction tilted by an angle $\psi$ with respect to the long bone axis (see eq. A3 for definition). A completely random orientation of needle-like crystals would correspond to $\mathrm{h}(\psi) \equiv 1$. Maxima at $\psi=0$ indicate that the crystals are predominantly oriented parallel to the bone axis. This tendency is less pronounced in oim/oim bone. 
Table I. Degree of Alignment $f_{a}$ Typical Tilt Angle $\psi_{a}$ and Average Thickness $T$ of the Mineral Crystals

\begin{tabular}{lccc}
\hline & $\mathrm{f}_{\mathrm{a}}$ & $\psi_{\mathrm{a}}$ & $\mathrm{T}$ \\
\hline & $\%$ & ${ }^{\circ} \mathrm{C}$ & $n m$ \\
oim/oim & & & \\
Femur 1 & 23 & 29 & 1.60 \\
Femur 2 & 37 & 22 & 1.73 \\
Tibia 1 & 30 & 26 & 1.68 \\
Tibia 2 & 37 & 22 & 1.81 \\
mean \pm sd & $32 \pm 7$ & $25 \pm 3$ & $1.71 \pm 0.09$ \\
+ /oim & & & \\
Femur 1 & 49 & 22 & 1.90 \\
Femur 2 & 41 & 22 & 1.83 \\
Tibia 1 & 40 & 24 & 1.84 \\
Tibia 2 & 43 & 22 & 1.93 \\
mean +sd & $43 \pm 4$ & $23 \pm 1$ & $1.88 \pm 0.05$ \\
& & &
\end{tabular}

According to a Student's $t$ test, $\mathrm{f}_{\mathrm{a}}$ and $\mathrm{T}$ are both significantly larger in heterozygotes than in homozygotes $(P<0.026$ and $P<0.015$, respectively). There could also be a tendency for a larger $\psi_{\mathrm{a}}$ in oim/oim, although the difference is not statistically significant. Taking the data for heterozygotes and homozygotes together, $\mathrm{f}_{\mathrm{a}}$ and $\mathrm{T}$ are positively correlated $\left(r=0.94, P<0.001\right.$, slope $\left.=67 \pm 10 \mathrm{~nm}^{-1}\right)$ as shown also in Fig. 7 . When the oim/oim data are considered separately, the regression analysis between $\mathrm{f}_{\mathrm{a}}$ and $\mathrm{T}$ gives the same slope and correlation coefficient $r=$ 0.93 , slope $=70 \pm 20 \mathrm{~nm}^{-1}$ ), but only $P<0.07$ due the small number of data points.

one would expect the distribution to be a horizontal line in Fig. 4. The measured distributions of crystal orientation are indicated in Fig. 4 by thick lines. The distribution is much more elongated in the horizontal direction for the heterozygote than for the oim/oim mouse.

One can further notice that, for all curves in Fig. 3, h $(\psi)$ approaches a constant at large angles $\left(\psi>60^{\circ}\right)$. This result could indicate the presence of a completely randomly oriented collagen/mineral composite (the amount which corresponds to the constant value of $\mathrm{h}(\psi)$ at $\psi>60^{\circ}$, which we call $\left.\mathrm{h}_{0}\right)$ together with material containing aligned crystals as represented by the peak in Fig. 3. Based on this interpretation, the "degree of alignment" may be defined as the fraction $f_{a}=1-h_{0}$ of crystals represented by the peak in Fig. 3 . The values for $f_{a}$ (in percent of total collagen/mineral composite) are given in Table I.

To determine the most frequent tilt angle within the distribution of aligned crystals, a plot of $\left(h(\psi)-h_{0}\right) \sin \psi$ may be derived. This is shown in Fig. 5. The maximum position of this distribution can be interpreted as the typical tilt angle $\psi_{\mathrm{a}}$ of the aligned crystals with respect to the long bone axis. The values for $\psi_{\mathrm{a}}$, typically around $20^{\circ}$ for the bones obtained from the heterozygote animal, values in agreement with those published in earlier studies on normal mice $(12,13,20)$, are somewhat larger in the oim/oim case and are given in Table I.

The spherically averaged SAXS intensity $J(q)$ from eq. A1 was used to determine the average crystal thickness T (12-15). The values obtained for $\mathrm{T}$ (given in Table I) are close to $2 \mathrm{~nm}$ for the heterozygote tissues (as indeed expected for young mice) (12) but are smaller for the oim/oim bones. The function $\mathrm{G}(\mathrm{x})$, characteristic of the shape of mineral crystals (14), was also determined but did not show a significant difference between oim/oim and heterozygote samples (Fig. 6). This means that in all cases the mineral crystals were very elongated objects, that is longer than the $\sim 50 \mathrm{~nm}$ resolution of the SAXS method, differing only by their average thickness. The $G(x)$ curve was also in good agreement with a theoretical model (15) assuming a distribution of needle-like crystals in bone tissue. It was, however, quite different from a $\mathrm{G}(\mathrm{x})$-curve based on human bone analysis and for which the tissues are compatible with a predominance of plate-like crystals (14).

Fig. 7 plots together a number of parameters derived for the present samples. It is apparent that there is a striking positive $(r=0.94, P<0.001)$ correlation between the degree of alignment $\mathrm{f}_{\mathrm{a}}$ and the average thickness $\mathrm{T}$ of the mineral crystals for both the oim/oim and heterozygote bones. This correlation even seems to hold for different locations in the bones of one homozygous animal. A correlation between the typical tilt angle of the crystals $\psi_{\mathrm{a}}$ and T is less obvious, but there could be a tendency (although not statistically significant, see Table I) for larger and more variable tilt angles in oim/oim bone as the crystal thickness becomes smaller.

\section{Discussion}

The mechanical strength and integrity of a calcified tissue lie in a number of different sources at various levels of its respective

\section{+loim oim/oim}

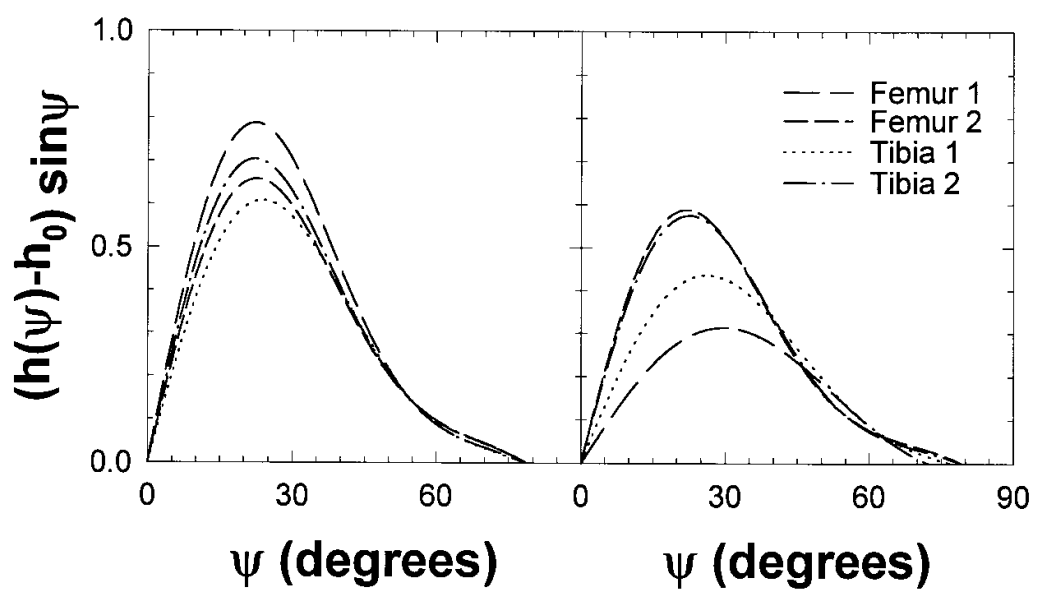

Figure 5. Angular distribution of the aligned mineral crystals. The function $\mathrm{h}(\psi)$ is shown in Fig. 3 and $h_{0}$ is the average value of $h(\psi)$ in the range $60^{\circ}$ $<\psi<90^{\circ}$. The maximum of each of the curves occurs at the position $\psi_{\mathrm{a}}$, the typical tilt angle of needle-shaped crystals with respect to the long bone axis. The area below each curve represents $f_{a}$, the degree of alignment. 


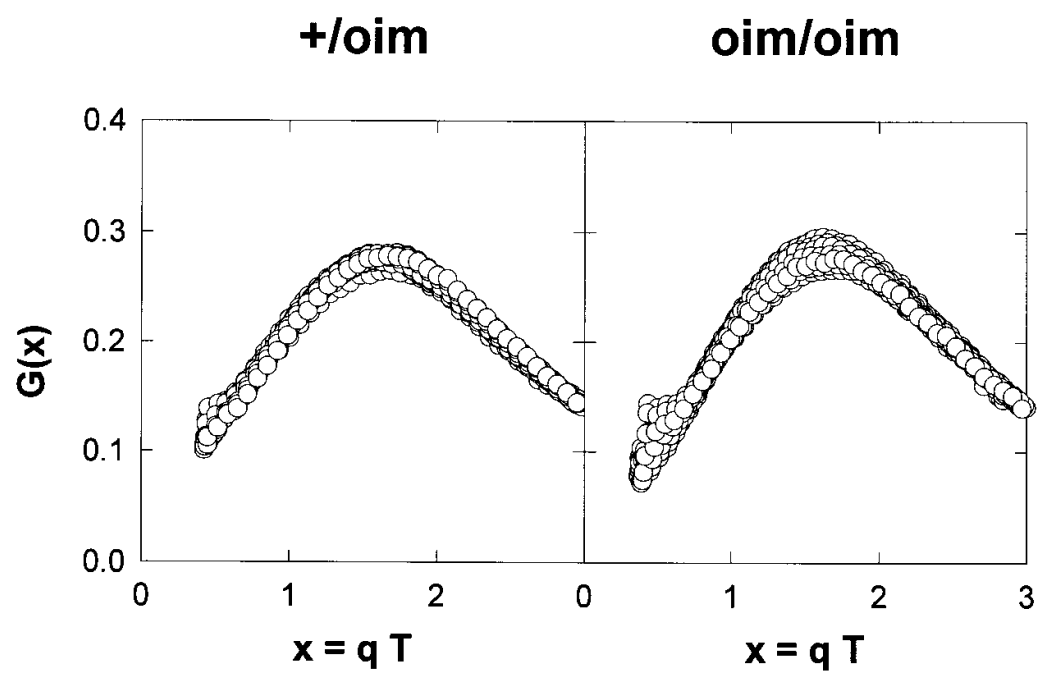

Figure 6. SAXS-shape function $\mathrm{G}(\mathrm{x})$ for the homozygous oim/oim and the heterozygous +/oim animal. Note that totals of 17 and 23 measurements at various positions in the femurs and tibiae are superimposed on the graphs for +/oim and oim/oim, respectively. There is a well-defined maximum in the graphs, in agreement with the existence of needlelike crystals in the bone tissues (12-15). Both graphs appear to be rather similar. The only difference occurs at very small $\mathrm{x}$, where contributions from crystals at the edge of the largest size accessible to SAXS (that is, all dimensions larger than $50 \mathrm{~nm}$ ) might explain the slight increase of $\mathrm{G}(\mathrm{x})$ at very small $\mathrm{x}$ in the case of some measurements in oim/ oim bone. Indeed, the presence of such large crystals has been revealed previously by electron microscopy $(10,11)$. structural hierarchy $(11,16)$. In part, these two parameters have been suggested to derive from a close, molecular interaction between the composite of mineral phase and the organic constituents with which the mineral associates (11). In this context of tissue stability, then, it is important to consider the physical and chemical features of both the inorganic and organic components. With regard to the former, the size and

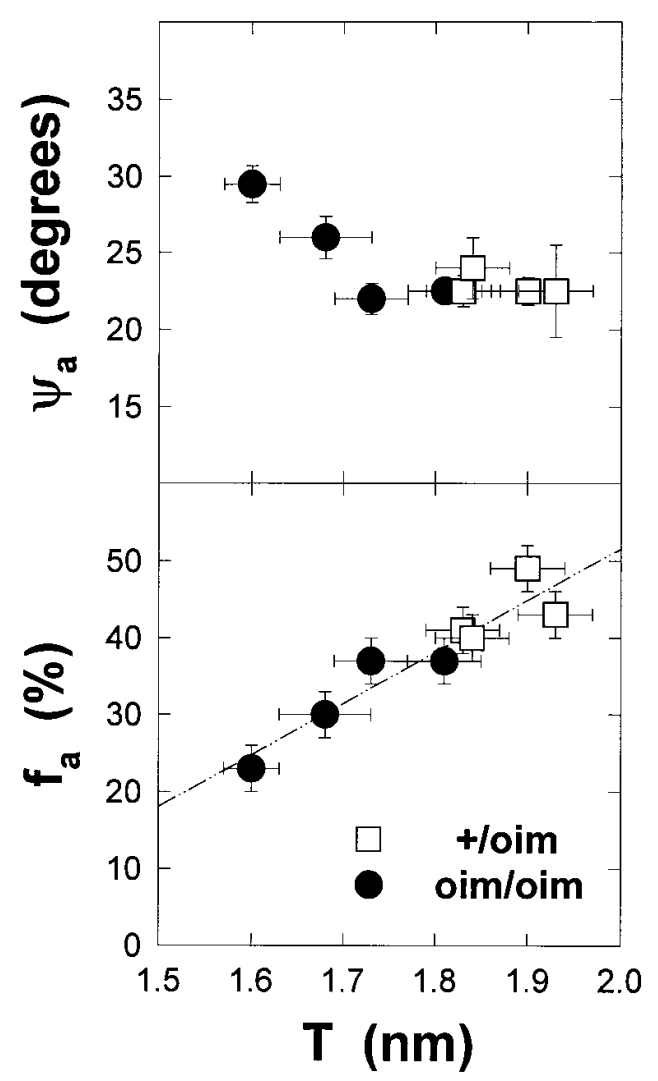

Figure 7. Typical tilt angle $\psi_{\mathrm{a}}$ and degree of alignment $\mathrm{f}_{\mathrm{a}}$ of needleshaped crystals plotted as a function of needle thickness T. The dashdotted line yields a linear correlation $(r=0.94, P<0.001)$ between $\mathrm{f}_{\mathrm{a}}$ and T. Errors in the measurements of the respective parameters are shown by individual error bars and represent one standard deviation of mean values. shape and the location, alignment, and orientation of the mineral crystals with respect to the organic matrix are fundamentally important (11). Some of these mineral characteristics have been examined in osteogenesis imperfecta in other studies $(11,21)$, but the nature of the mineral features could not be quantitated in such investigations to the same extent as it can be using SAXS (12-15). Here, the degree of alignment, tilt (orientation), and thickness of the mineral crystals have been determined for the first time by SAXS in the cortical bone from the midshaft region of femurs and tibiae of an osteogenesis imperfecta mouse model and its heterozygous counterpart. The results may be appropriate to understanding the basis for the observed brittleness in this and other tissues affected by the disease.

The principal results of the comparison between the homozygous oim/oim mouse and its +loim counterpart can be summarized as follows. First, despite the fact that collagen in oim/oim consists exclusively of $\alpha 1(\mathrm{I})$ homotrimers, there is some similarity in the general shape of the cortical bone crystals from homozygous and heterozygous animals and also from normal mice investigated in earlier experiments $(13,15)$. It should be noted that the heterozygotes would be expected to have a $\sim 50 \%$ composition of homotrimers (22), although we are not aware of any direct measurement of this parameter. Second, the mineral crystals within the oim/oim bone are thinner than in the heterozygous littermate, and also thinner than in normal mice of a corresponding age (13). Such an observation is consistent with the suggestion that the assembly and organization of collagen homotrimers in oim/oim may critically affect the volume, stereochemistry, or electrochemistry that is required to accommodate mineral formation at molecular and higher order structural levels and lead to crystals more blocklike than normal in some instances (inside tendons) $(10,11)$ and longer and narrower in others, as found here for cortical bone of femur or tibia. Third, the degree of alignment of the crystals with respect to the long axis of the bones is more variable in the oim/oim animal. This observation agrees with electron diffraction results showing crystals both aligned with the long axis of collagen and generally randomly oriented in the mineralized tendons from oim/oim mice (11). The variability of crystal alignment in affected bone may also be a consequence of the putative changes in volume or other structural characteristics dictated by altered intra- or intermolecular and 
higher order collagen structure $(9,11)$. Fourth, both the bone crystal thickness and the degree of alignment are more variable in the homozygous animal compared to the heterozygote but both these parameters are closely correlated.

If one puts these results together, oim/oim bone can be supposed to consist of a heterogeneous mixture of regions of roughly normal collagen/mineral composite, although containing thinner and less well aligned crystals, and regions having relatively larger (all dimensions exceeding $50 \mathrm{~nm}$ ), block-like mineral deposits. The latter are too large to be resolved by SAXS but have been well documented in other work using electron microscopic tomography of calcifying tendons in the same oim/oim mice as examined here $(10,11)$. Heterogeneity in bone mineral has also been found in human OI by electron microscopy (4), which revealed the coexistence of apparently normal lamellar bone regions with other regions containing apatite crystals deposited on surfaces of abnormal collagen fibrils.

In the light of the present SAXS data, the collagen/mineral composite in oim/oim bone resembles the bone from healthy but much less developed mice. Because of the close correlation between the two parameters $f_{a}$ and T (Fig. 7), one might speculate that the mineralization was equivalently delayed in oim/ oim, thus making the mineralized bone appear less mature. Whatever the character of the affected tissue, it may be related to the recent report that the molecular spacing in collagen fibrils from oim/oim mice is larger than normal and that there is no apparent quasihexagonal lattice in collagen packing (B. Brodsky, personal communication) (for a recent discussion of the molecular ordering in normal collagen fibrils see, for example, Hulmes et al. [23]). Hence, one may speculate that the increased molecular spacing and reduced packing order of OItype fibrils might allow greater variability in crystal alignment and could be an explanation for the increased tilt angle $\psi_{\mathrm{a}}$ observed here in oim/oim bone. It is also possible, as suggested very recently (11), that the collagen defect consists of multiple axial misregistrations of the collagen molecules comprising the fibrils, leading to limited volumes within contiguous hole zones, instead of the putative large channels in which mineral crystals would normally nucleate and develop. The resulting crystals would lengthen as usual with little change in their width and thickness as they elongated and as such in agreement with the results of the present work.

In conclusion, the present data give clear evidence that the mineral crystals are thinner and less well aligned in cortical oim/oim bone, a result expected to reduce the biomechanical quality of the tissue. Together with the heterogeneous mineralization defects described in earlier electron microscopy work $(4,10,11)$, this could contribute to the extreme brittleness of bone in osteogenesis imperfecta.

\section{Appendix}

The SAXS intensity as shown in Fig. 1 is given as $\mathrm{I}(\mathbf{q})$, with the horizontal component $\mathrm{q}_{\mathrm{x}}$ of the scattering vector $\mathbf{q}$ parallel to the major axis of the long bone and the vertical component $\mathrm{q}_{\mathrm{y}}$ perpendicular to it. The vector $\mathbf{q}$ may also be written in polar coordinates as $(\mathrm{q}, \phi)$, where $\tan (\phi)=q_{\mathrm{x}} / q_{\mathrm{y}}$ and $q=\sqrt{q_{\mathrm{x}}{ }^{2}+q_{\mathrm{y}}{ }^{2}}$. The length $\mathrm{q}$ of $\mathbf{q}$ is related to the scattering angle $\theta$ between the incident and the diffracted beam by $q=(4 \pi / \lambda) \sin (\theta / 2)$. To determine $h(\psi)$, one may separate the intensity into a $\phi$ - and a q-dependent term $(18,19)$ :

$I(\boldsymbol{q})=I(q, \phi)=J(q) R(\phi)$, where
$J(q)=\int_{0}^{\pi / 2} I(q, \phi) \cos (\phi) d \phi$

is the spherically averaged intensity and where

$R(\phi)=4 \int_{\phi}^{\pi / 2} \frac{h(\psi) \sin \psi}{\sqrt{\cos ^{2} \phi-\cos ^{2} \psi}} \mathrm{d} \psi$,

with the convention that $\int_{0}^{\pi / 2} h(\psi) \sin (\psi) d \psi=1$. The parameter $\mathrm{J}(\mathrm{q})$ has been determined (see eq. A2) from the original SAXS data by integrating $\mathrm{I}(\mathrm{q}, \phi)$ with respect to $\phi$, and $\mathrm{S}(\phi)=\rho \mathrm{R}(\phi)$ by integrating it with respect to q (see eq. A1). The constant $\rho$ depends on the experimental setup and is irrelevant for the present purpose. By inverting equation (3), it is possible to determine the angular distribution $\mathrm{h}(\psi)$ of the elongated mineral crystals. This may be done, in principle, by numerical integration (18). Since $\mathrm{R}(\phi)$ is only known with limited precision from the SAXS measurements, a simpler procedure may be used by fitting the function\&

$$
S(\phi)=k \cos ^{\mathrm{m}} \phi+1 \cos ^{\mathrm{n}} \phi,
$$

to the experimentally determined $\mathrm{S}(\phi)$, the constants $\mathrm{k}, 1, \mathrm{~m}$, and $\mathrm{n}$ being the fit parameters. One can then show theoretically that the corresponding function $\mathrm{h}(\psi)$ may be expressed as

$$
\begin{gathered}
h(\psi)=\frac{k(m+1) u_{\mathrm{m}} \cos ^{\mathrm{m}} \psi+1(n+1) u_{\mathrm{n}} \cos ^{\mathrm{n}} \psi}{k u_{\mathrm{m}}+1 u_{\mathrm{n}}}, \\
\text { where } u_{\mathrm{m}}=\Gamma\left(\frac{m+2}{2}\right) / \Gamma\left(\frac{m+3}{2}\right)
\end{gathered}
$$

The symbol $\Gamma$ indicates the Gamma-function.

\section{Acknowledgments}

We are grateful to Dr. B. Brodsky (Dept. of Biochemistry, UMDNJ, Piscataway, NJ) for discussions and for communicating results on collagen packing in tendon from oim/oim animals prior to publication.

O. Paris is supported by the Fonds zur Förderung der Wissenschaftlichen Forschung in Austria (Proj. S5601). W. J. Landis acknowledges support from the National Institutes of Health (grants AR-41452, AR-34078, and AR-34081).

\section{References}

1. Kuivaniemi, H., G. Tromp, and D. J. Prockop. 1991. Mutations in collagen genes: causes of rare and some common diseases in humans. FASEB J. 5: 2052-2060.

2. Byers, P. H., G. A. Wallis, and M. C. Willing. 1991. Osteogenesis imperfecta: translation of mutation to phenotype. J. Med. Genet. 28:433-442.

3. Cole, W. G. 1994. Osteogenesis imperfecta as a consequence of naturally occurring and induced mutations of type I collagen. Bone Miner. Res. 8:167204.

4. Traub, W., T. Arad, U. Vetter, and S. Weiner. 1994. Ultrastructural studies of bone from patients with osteogenesis imperfecta. Matrix. 14:337-345.

5. Vetter, U., M. A. Weis, M. Mörike, E. D. Eanes, and D. R. Eyre. 1993. Collagen crosslinks and mineral crystallinity of patients with osteogenesis imperfecta. J. Bone Miner. Res. 8:133-137.

6. Cassella, J. P., and S. Y. Ali. 1992. Abnormal collagen and mineral formation in osteogenesis imperfecta. Bone Miner. 17:123-128.

7. Stöss, H., and P. Freisinger. 1993. Collagen fibrils of osteoid in osteogenesis imperfecta: morphometrical analysis of the fibril diameter. Am. J. Med. Gen. 45:257.

8. Chipman, S. D., H. O. Sweet, D. J. McBride, Jr., M. T. Davisson, S. C. Marks, Jr., A. R. Shuldiner, R. J. Wenstrup, D. W. Rowe, and J. R. Shapiro. 1993. Defective prox2(I) collagen synthesis in a new recessive mutation of mice: A model of human osteogenesis imperfecta. Proc. Natl. Acad. Sci. USA. 90:1701-1705.

9. Deleted in proof.

10. Landis, W. J. 1995. Tomographic imaging of collagen-mineral interaction: Implications for osteogenesis imperfecta. Connect. Tissue Res. In press. 
11. Landis, W. J. 1995. The strength of calcified tissue depends in part on the molecular structure and organization of its constituent mineral crystals in their organic matrix. Bone 16:533-544.

12. Fratzl, P., N. Fratzl-Zelman, K. Klaushofer, G. Vogl, and K. Koller. 1991. Nucleation and growth of mineral crystals in bone studied by SAXS. Calcif. Tissue Int. 48:407-413.

13. Fratzl, P., M. Groschner, G. Vogl, H. Plenk, J. Eschberger, N. FratzlZelman, K. Koller, and K. Klaushofer. 1992. Mineral crystals in calcified tissues: a comparative study by small-angle x-ray scattering. J. Bone Miner. Res. 7: 329-334.

14. Fratzl, P., P. Roschger, J. Eschberger, B. Abendroth, and K. Klaushofer. 1994. Abnormal bone mineralization after fluoride treatment in osteoporosis: a small-angle x-ray scattering study. J. Bone Miner. Res. 9:15411549.

15. Fratzl, P. 1994. Statistical model for the habit and arrangement of mineral crystals in the collagen of bone. J. Stat. Phys. 77:125-143.

16. Wagner, H. D., and S. Weiner. 1992. On the relationship between the microstructure of bone and its mechanical stiffness. J. Biomech. 25:1311-1320.

17. Landis, W. J., M. C. Paine, and M. J. Glimcher. 1977. Electron micro- scopic observations of bone tissue prepared anhydrously in organic solvents. $J$. Ultrastruct. Res. 59:1-30.

18. Perret, R., and W. Ruland. 1969. Single and multiple x-ray small-angle scattering of carbon fibres. J. Appl. Cryst. 2:209-218.

19. Matsushima, N., M. Akiyama, and Y. Terayama. 1982. Quantitative analysis of the orientation of mineral in bone from small-angle $\mathrm{x}$-ray scattering patterns. Jap. J. Appl. Phys. 21:186-189.

20. Landis, W. J., M. J. Song, A. Leith, L. McEwen, and B. McEwen. 1993. Mineral and organic matrix interaction in normally calcifying tendon visualized in three dimensions by high-voltage electron microscopic tomography and graphic image reconstruction. J. Struct. Biol. 110:39-54.

21. Vetter, U., E. D. Eanes, J. B. Kopp, J. D. Termine, and P. Gehron Robey. 1991. Changes in apatite crystal size in bones of patients with osteogenesis imperfecta. Calcif. Tissue Int. 49:248-250.

22. Dunn, M. G., D. J. McBride, and J. R. Shapiro. 1995. Absence of the collagen $\alpha 2$ (I) chain diminishes the mechanical properties of bone. Trans. Or thop. Res. Soc. 20:153.

23. Hulmes, D. J. S., T. J. Wess, D. J. Prockop, and P. Fratzl. 1995. Radial packing, order and disorder in collagen fibrils. Biophys. J. 68:1661-1670. 